\section{Stay where you put them}

Trycare Ltd is the UK distributor for Adin Dental Implant Systems - the world's tenth largest manufacturer of dental implants. Clinical tests in universities and hundreds of thousands of successful restorations make Adin implants the first choice for many implantologists.

Adin's spiral design implants are suitable for all indications and are exceptional for immediate loading. Their surfaces have been treated for faster osseointegration and New York University research indicates that they integrate as well as Nobel, Straumann and Astra.

Their unique thread and tip slices through bone compressing and condensing on the way, offering outstanding primary stability and optimal restorative orientation, while their surface treatment has been shown to increase osseointegration. Macroscopic grooves on the surface of the implant thread double the surface area of most other implants and give better bone-to-implant contact.

\section{A new, cutting edge option for older patients}

Solvay Dental 360 has developed a new generation polymer called Ultaire AKP specifically for the fabrication of metalfree removable partial dentures.

This material is an ideal alternative because it is strong and offers stable retention, but is also lightweight and biocompatible.

As the manufacturers of Ultaire AKP, Solvay Dental 360 can also arrange a professional 'Lunch and Learn' session at your dental practice to show you and your team the benefits of digitally designed, polymer-based RPD frameworks.

To find out more about Solvay Dental 360, Ultaire AKP and Dentivera milling discs, please visit www.solvaydental360.com.
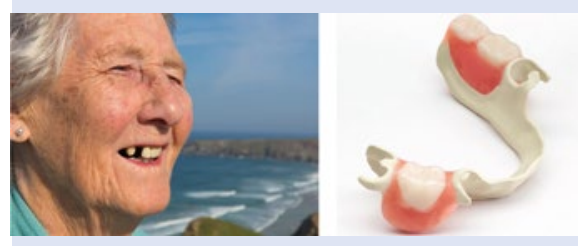

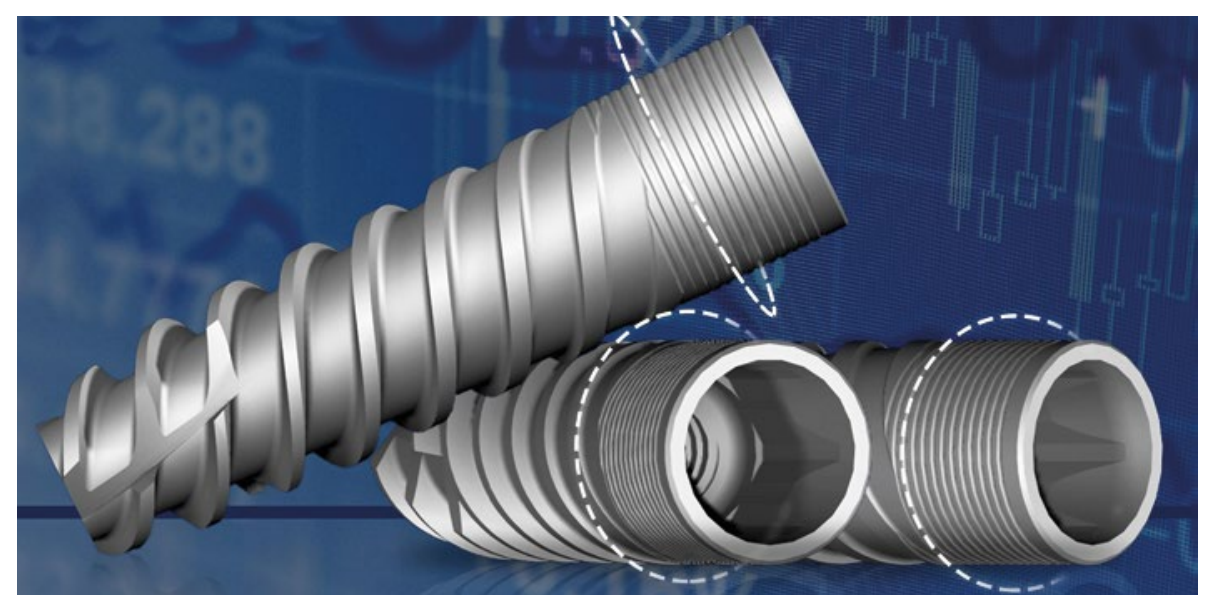

Adin spiral design implants can be redirected during placement with little or no reduction of stability, the dual cutting blade edge helping to minimise the osteotomy site and trauma to bone and surrounding soft tissue. This makes them ideal for thinner bone ridges.

Adin implants are the next-generation of bone-level implants and one of the highest performing implants available. They are a favourite with many of the FGDP implant course tutors at The Royal College of Surgeons.

For new users, Trycare Ltd offer a range of introductory offers designed to help clinicians take advantage of Adin implants' unique benefits.

More information is available at www. trycare.co.uk/adin, or request a Surgical Essentials and Adin catalogue by contacting your local representative or calling 01274 885544.

\section{Hassle-free ordering}

As a first-class supplier of high quality dental products, Denka prides itself on offering a hassle-free service that is designed to meet the needs of modern practices.

With a catalogue of more than 1,000 dental products from many of the industry's leading brands, Denka says it remains dedicated to delivering competitively priced supplies for both general and specialist dentistry.
These supplies include consumable items, disinfectants, crown-cutting burs, endodontic materials, restorative solutions, implant systems, and much more.

Using the DenkaSMART supplier portal, practices can take advantage of an automatic inventory management system for reduced risk of under or over ordering.

More information is available by visiting www.denkauk.com calling 08007076212 or emailing hello@denkauk.com.

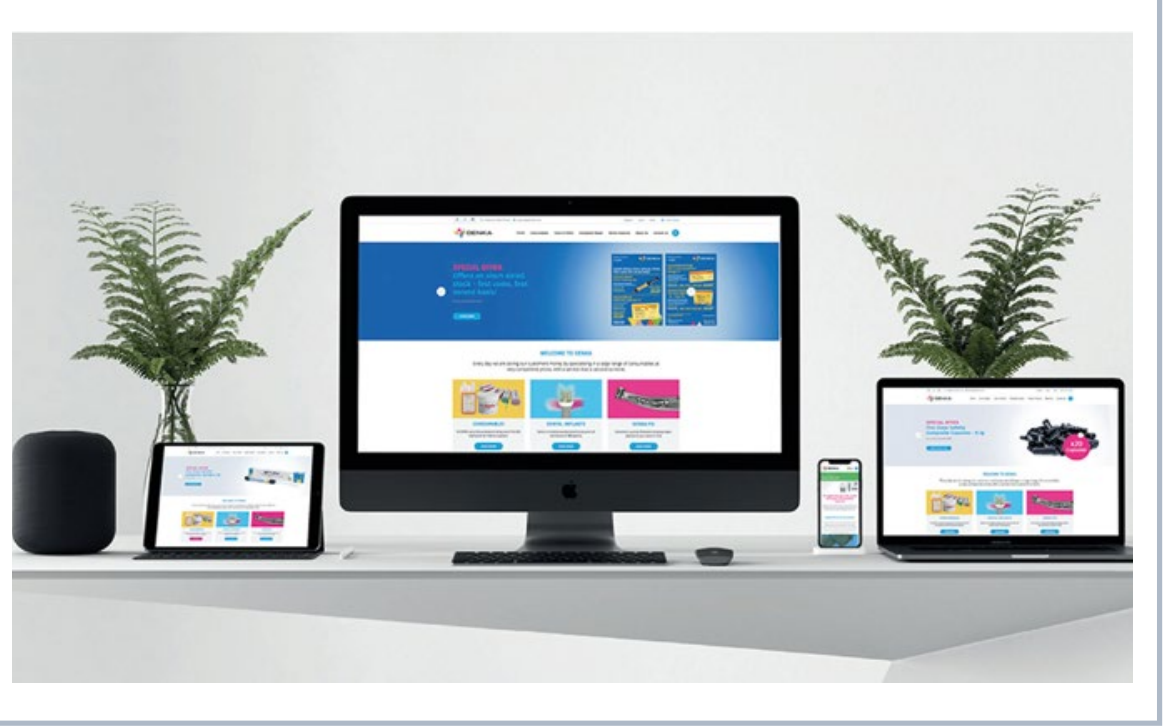

